\title{
A mathematical interpretation and validation of the Streamline's shape theory for inviscid- incompressible flows \& viscid-compressible flows of the Newtonian fluids
}

\author{
Yuvaraj George
}

\author{
To my friend B. Pavan Kumar Reddy
}

\begin{abstract}
This article objectively assesses, the hypothesis of the streamline's shape theory and its formulated equation. The deduction of proof uses algebra rather than first-order partial differential equations to address the specific hypothesis of "Streamline's shape theory" from the fundamental perspective of applied mathematics and scientifically derives mathematical relations of the axioms and corollaries in the field of fluid dynamics. The algebraic methods employed provide progressively more distinct and precise solutions compared to first-order partial differential equations. The foremost objective of this work is to evaluate if the formulations of the streamline's shape theory can have solutions for inviscid-incompressible and viscid-compressible flows of Newtonian fluids and to identify their nature. Secondly, to understand how the topology of the body and the free-stream conditions affect these solutions with due regards to the shape and size of the body interacting with the fluid flow. Finally, to explore the possibility of this theory to develop a CFD solver for streamline simulation to reduce the experimentation in the analysis of flow-structure interactions of Newtonian fluids and also to identify its scope of applications and limitations.
\end{abstract}

Mathematics Subject Classification (2010). 35Q35 ; 74F10 ; 76M99.

Keywords. Fluid-Structure interactions, Topological fluid dynamics, General fluid mechanics, Mathematical fluid dynamics, Applied mathematics.

\section{Introduction}

The existing theories concerning streamlines like Prandtl-Batchelor theorem and free streamline theory were fundamental in the development of several 
models for the streamline simulation. Prandtl-Batchelor theorem is applicable for flows with high Reynolds numbers for closed streamline conditions and invalid within the boundary layer regime. 1] Freestream line theory, on the other hand, uses classical Helmholtz free boundary theory and Laplace transformations. Based on it, few promising mathematical models were developed in recent years for various complex cases in hydrodynamics and aerodynamics, such as wake models, cavity flows, free-surface perturbations caused by viscosity, and turbulence. [2, 3, 4, 4, 5, 6, 7, 8] Historically, many theories use energy conservation/ dissipation and mass conservation/ dissipation approaches. These approaches give an aggregate of fluid flow within the considered boundary limits. However, they present a challenge to specify local parameters within the fluid flow. Different types of solvers use different methods and equations concerning various cases of the fluid flow and types of fluids. Most of them employ differential equations and partial differential equations to evaluate local parameters that have their shortcomings. In recent years streamline simulation has acquired its prominence among researchers as a promising approach compared to traditional methods. [9] Moreover, the reliability of CFD results heavily depends on the computational methods, basic equations, and processing capabilities of the computer, and thus experimental data is always required. [10]

Though existing literature conveys that streamlines form as a result of shear stress and dynamic pressure distributions over the surface of the body in a flow. 11] There efficiently is no mathematical model that can explain how and why streamlines are forming concerning the nature of fluid, nature of the flow, the shape, and the size of the body.[12, 13 .

"Streamline's shape theory" is a proposed mathematical model that claims to explain the mechanism of streamlines of a Newtonian fluid in an open circuit flow over a body. According to this theory,

Hypothesis: Laminar flow of a Newtonian fluid over a rigid body will displace the fluid particles when the solid body has sufficient strength to withstand the deformation and displacement. The dynamic pressure of the fluid and the shear stress distribution over the body contributes to the streamline formation. [14, 12]

Equation (1) shows the force that is resultant of flow over a rigid body, which contributes to change in the trajectory of fluid particles forming streamlines.

$$
\begin{aligned}
\vec{F}_{A_{i}}= & \oiint_{A}\left(\frac{1}{2} \rho_{A_{i}}{\overrightarrow{\vec{A}_{i}}}^{2}+\frac{1}{2} \rho_{A_{i-1}}{\overrightarrow{v_{A_{i-1}}}}^{2} \cos \left(\theta_{r}\right)\right) d A \\
& +\oiint_{A}\left(\mu_{A_{i}} \frac{\partial v_{A_{i}}}{\partial y}+\mu_{A_{i-1}} \frac{\partial v_{A_{i-1}}}{\partial y} \cos \left(\theta_{r}\right)\right) d A
\end{aligned}
$$

Here, equation (1) is the sum of dynamic pressure and shear stress at a local point and the impact of surrounding points of the topology. The $\frac{1}{2} \rho_{A_{i}} v_{A_{i}}{ }^{2}$ is dynamic pressure at a local area $A_{i}$ and $\mu_{A_{i}} \frac{\partial v_{A_{i}}}{\partial y}$ is the shear 
stress at $A_{i}$. Similarly, the cosine multiples in the equation (1.1), are dynamic pressure and shear stress at surrounding areas and $\theta_{r}$ is the angle between free-stream vector and the local force vector. $\theta_{r}$ is the angle at which the resultant force $F_{A_{i-1}}$ acts on $F_{A_{i}}$.

Remark 1.1. The aforementioned mathematical formula claims to predict and explain the trajectory of fluid particles in a laminar flow of Newtonian fluid and provides an ability to explain various phenomena in an open circuit flow such as stalling, flow separation, down-wash, Coand $\breve{a}$ effect, effects of shape and size of the body over which fluid flows. Though the theory is argumentatively not disapproved but its reliability in terms of mathematical precision in analyzing open circuit Newtonian flow over a body is yet to be evaluated.

That imposes certain questions like: Does this equation always have solutions? If so, what is the nature of the resultant solution? What is the range of applicability for this formula?

\section{Methodology}

The article published under the title "Mechanics of streamlines in an open circuit fluid flow and its impact on the flow" [12] is a mathematical conjecture as it doesn't have any mathematical proof or experimental data to validate it.

A brief mathematical inference for the stated formulation of "Streamline's shape theory," is carried out in section 3. Then, the mathematical proof employing inductive logic and the direct method of proof derives mathematical inferential arguments in the field of fluid mechanics. The section 4 titled "mathematical validation" has both graphical method and analytical methods of algebraic calculations that proves the formulation of the considered hypothesis of "Streamline's Shape Theory."

\section{Mathematical Interpretation}

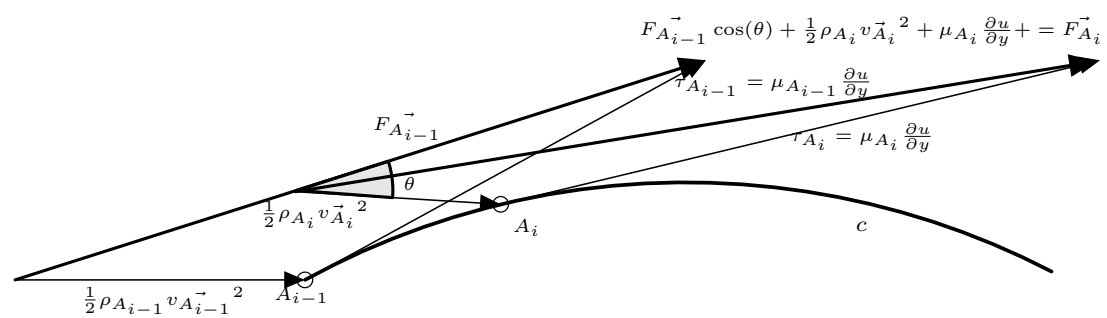

Figure 1. Relationship between two successive points on the topology of the body and the vectors due to dynamic pressure and shear stress distribution 
The shear stress always acts tangential to the surface of the body for a Newtonian fluid, and dynamic pressure acts normal to the surface of the body. 115] Equation (1) is a time-independent and sum of two parabolic functions and two linear functions.

Here, dynamic pressure at $A_{i}$ and resultant dynamic pressure from surrounding areas $A_{i-1}$ are the parabolic functions as they are in the form: $a x^{2}=\frac{\rho}{2} \vec{v}^{2}$. The shear stress distribution is a linear function for a Newtonian fluid within subsonic velocities. The $\mu \frac{\partial v}{\partial y}$ is in the form of $m x+0$. As the fluid stream passes over the topology of a body, the shear stress distribution and the dynamic pressure distribution change the velocity and trajectory of the fluid particles. Therefore, a free-stream fluid flow, a defined parabolic function, when passes over a body develop shear stress on the topology of that body, which is a linear function for the Newtonian fluids. The sum of these two functions will have a resultant force vector, which effects succeeding locus of points gradually.

Figure.1 illustrates these vectors. The dynamic pressure due to freestream at $\frac{1}{2} \rho_{A_{i-1}} \vec{v}_{A_{i}}^{2}$ and the shear stress developed at $A_{i-1}$ is $\mu_{A_{i-1}} \frac{\partial v_{A_{i-1}}}{\partial y}$ and the resultant sum due to these two forces is $F_{A_{i-1}}$. The force on the succeeding area $A_{i}$ along the path 'c' is a sum of $F_{A_{i-1}}$, the force due to the dynamic pressure of free-stream, and the force due to the shear stress distribution. Here, $A_{i-1}$ is the stagnation point. So, the formation of streamline starts at the stagnation point and gradually changes along with the shape and size of the body.

\section{Mathematical Validation}

The algebraic form of the equation (1) at any point on the surface of the body with arbitrary shape is: [16]

$\frac{1}{2} \rho_{A_{i}} v_{A_{i}}{ }^{2}+\mu_{A_{i}} \frac{v_{A_{i}}}{y}+\frac{1}{2} \rho_{A_{i-1}} v_{A_{i-1}}^{2} \cos \left(\theta_{r}\right)+\mu_{A_{i-1}} \frac{v_{A_{i-1}}}{y} \cos \left(\theta_{r}\right)+P_{A_{i}}=0$

Here, $P_{A_{i}}$ is the pressure at $A_{i}$ and $\frac{v}{y}$ is the rate of shear deformation.

\subsection{In-viscid \& In-compressible flow}

For an inviscid-incompressible flow, $\rho$ and $\mu$ are constants. Since equation (2) is the sum of algebraic equations.Upon simplification,

$$
\underbrace{\frac{1}{2} \rho{\overrightarrow{A_{i}}}^{2}+\frac{\mu}{y}{\overrightarrow{A_{i}}}_{i}+q P_{A_{i}}}_{a x_{1}^{2}+b x_{1}+c}+\underbrace{\frac{1}{2} \rho{\overrightarrow{A_{i-1}}}^{2} \cos \left(\theta_{r}\right)+\frac{\mu}{y} v_{A_{i-1}} \cos \left(\theta_{r}\right)+r P_{A_{i}}}_{a x_{2}^{2}+b x_{2}+c}=0
$$

where, $\mathrm{q}+\mathrm{r}=1$ i.e. $q P_{A_{i}}+r P_{A_{i}}=P_{A_{i}}$ and $y$ is the length of the boundary layer formed. The $\frac{v}{y}$ is the rate of shear deformation. The sum of a parabolic function and linear function results in parabola shifting. Here, equation (3) is the sum of two algebraic functions. [17] Therefore, $f\left(x_{1}\right)+g\left(x_{2}\right)$ is the resultant function. Figure. 2 and figure. 3 graphically illustrates the algebraic sum of these two 
functions. Here, $\frac{1}{2} \rho \vec{v}^{2}$ and $\frac{1}{2} \rho \vec{v}^{2} \cos \left(\theta_{r}\right)$ are in the form of $a x^{2}$ and for $a>0$ the parabola will open upwards and will have a vertex at $(0,0)$. The vertex of the function $\frac{1}{2} \rho \vec{v}^{2}+\frac{\mu}{y} v+q P_{A_{i}}$ is $\left(-\frac{\nu}{y}, \frac{\mu \nu}{y}\left(\frac{\nu}{2 y}-1\right)+q P_{A_{i}}\right)$ and the vertex of $\frac{1}{2} \rho v_{A_{i-1}}^{2} \cos \left(\theta_{r}\right)+\frac{\mu}{y} v_{A_{i-1}} \cos \left(\theta_{r}\right)+r P_{A_{i}}$ is $\left(-\frac{\nu}{y}, \frac{\mu \nu}{y} \cos \theta_{r}\left(\frac{\nu}{2 y}-1\right)+r P_{A_{i}}\right)$. The $\mathrm{Y}$-intercept of the equation $(3)$ is $\left(0, P_{A_{i}}\right)$.

(a)Plot for the Velocity and Pressure at a local point of the flow for $\mathrm{f}\left(x_{1}\right)$

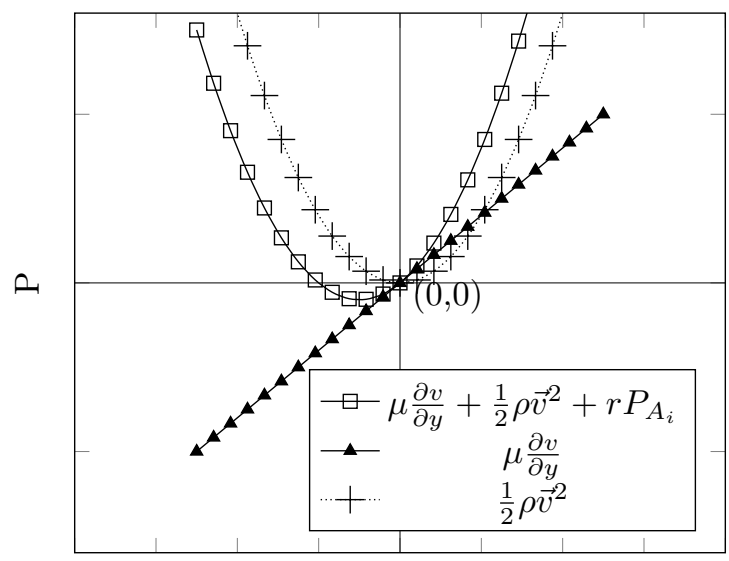

$\vec{v}$

(b)Plot for the Velocity and Pressure at a local point of the flow for $\mathrm{g}\left(x_{2}\right)$

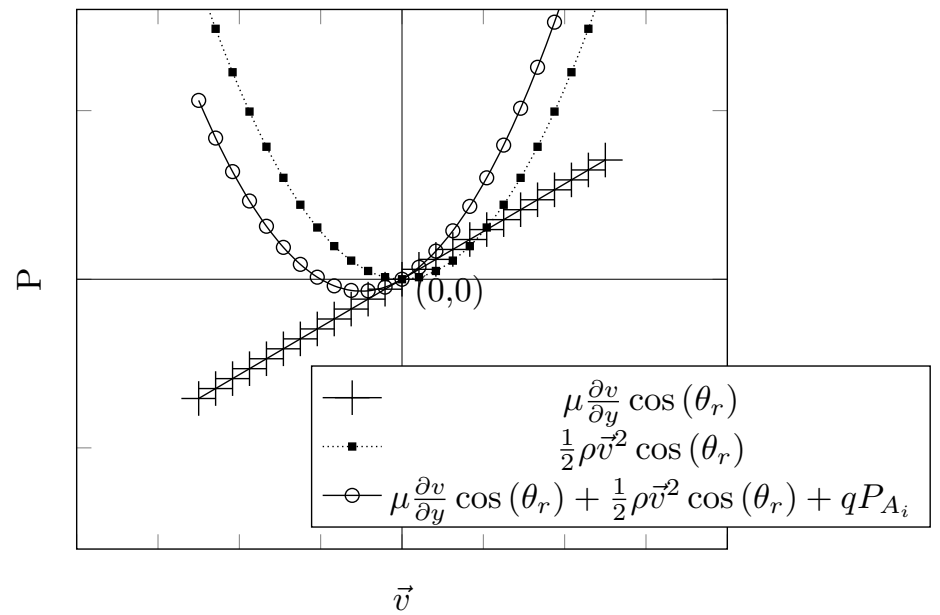

FiguRE 2. Graphical representation of the algebraic sum of dynamic pressure \& shear stress of the $f\left(x_{1}\right) \& g\left(x_{2}\right)$

As the negative sign indicates the only directional component of the velocity vector, the minimum velocity required for a fluid particle to change its 
Plot for the Velocity and Pressure at a local point of the flow for $f\left(x_{1}\right)+g\left(x_{2}\right)$

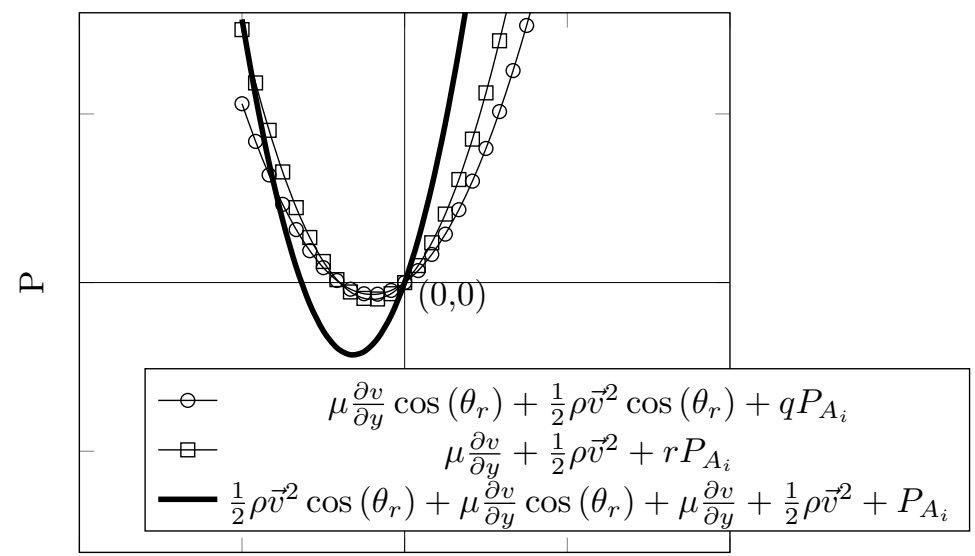

$\vec{v}$

FiguRE 3. Graphical representation of algebraic sum of the $f\left(x_{1}\right) \& g\left(x_{2}\right)$

trajectory and deform at a surface is $|-\nu / y|$. Thus, the corollary of kinematic viscosity is satisfied.

Corollary 4.1. For any change, in the fluid stream over a body. The minimum velocity after the interaction of a body with the fluid stream must be $\frac{\nu}{y}$. As Kinematic Viscosity is the inherent viscosity of Newtonian fluids, that does not change with a change in applied force.

Muller's method is a root-finding numerical method developed by David Muller that calculates roots. The standard formula where the co-efficient $a$, will be in the denominator and that leads to in-determinant solutions for the algebraic equation when $a=0$. Muller's formula can be deduced from the standard quadratic formula by Vieta's formulas, and it will give solutions even when $a=0$. The solution for $f\left(x_{1}\right)+g\left(x_{2}\right)$ will have four roots. The discriminants $\Delta_{i}$ and $\Delta_{i-1}$ of the functions $f\left(x_{1}\right)$ and $g\left(x_{2}\right)$ are given in the equation (4) and equation (5). Likewise, equation (6) gives the roots of the equation (3) by using Muller's method.[18 The increments and decrements of the resultant algebraic function depends on the value of cosine value in $g\left(x_{2}\right)$. As $\theta_{r}$ changes from $0^{\circ}$ to $360^{\circ}$ the value of $\cos \theta_{r}$ varies between 1 to -1 . This indicates the effects of the shape of the body on the flow. Therefore, the nature of the two roots for the considered algebraic expression depends on the shape of the body.

$$
\Delta_{i}=\left(\frac{\mu}{y}\right)^{2}-2 \rho\left(q P_{A_{i}}\right)
$$




$$
\begin{gathered}
\Delta_{i-1}=\left(\frac{\mu}{y} \cos \left(\theta_{r}\right)\right)^{2}-2 \rho\left(r P_{A_{i-1}}\right) \cos \left(\theta_{r}\right) \\
\overrightarrow{v_{i r}}=\frac{2 q P_{A_{i}}}{-\left(\frac{\mu}{y}\right) \pm \sqrt{\Delta_{i}}} ; \\
v_{i r-1}=\frac{2 r P_{A_{i}}}{-\left(\frac{\mu}{y}\right) \pm \sqrt{\Delta_{i-1}}}
\end{gathered}
$$

\subsection{Viscid- Compressible flow}

For a viscid compressible flow, $\rho$ and $\mu$ are not constants. Therefore, modifying equation (3) and introducing $\nu$, kinematic viscosity.

$$
\underbrace{\frac{1}{2} v_{A_{i}}^{2}+\frac{\nu}{y} v_{A_{i}}+\frac{q P_{A_{i}}}{\rho_{A_{i}}}}_{a x_{1}^{2}+b x_{1}+c}+\underbrace{\frac{1}{2} v_{A_{i-1}}^{2} \cos \left(\theta_{r}\right)+\frac{\nu}{y} v_{A_{i-1}} \cos \left(\theta_{r}\right)+\frac{r P_{A_{i}}}{\rho_{A_{i-1}}}}_{a x_{2}^{2}+b x_{2}+c}=0
$$

Since $\mu / y=\nu$ and $\nu$ is an inherent characteristic of the fluid which doesn't change with applied force.

Equation (7) is in the form of $a x_{1}^{2}+b x_{1}+c_{1}+a x_{2}^{2}+b x_{2}+c_{2}$ i.e $f\left(x_{1}\right)$ and $g\left(x_{2}\right)$. Similar to the method mentioned in the section 4.1, There will be a parabola shifting and change in the vertex of the algebraic expression. The vertex of the function $\frac{1}{2} v_{A_{i}}^{2}+\frac{\nu}{y}{\overrightarrow{A_{i}}}_{i}+\frac{q P_{A_{i}}}{\rho_{A_{i}}}$ is $\left(\frac{-\nu}{y},-1.5(\nu / y)^{2}+\frac{q P_{A_{i}}}{\rho_{A_{i}}}\right)$ and the vertex of $\frac{1}{2} v_{A_{i-1}}^{2} \cos \left(\theta_{r}\right)+\frac{\nu}{y} v_{A_{i-1}} \cos \left(\theta_{r}\right)+\frac{r P_{A_{i}}}{\rho_{A_{i-1}}}$ is $\left(\frac{-\nu}{y},-1.5(\nu / y)^{2} \cos \theta_{r}+\frac{r P_{A_{i-1}}}{\rho_{A_{i-1}}}\right)$. Similar to the method mentioned in section 4.1, the discriminants and roots of equation (7) are agiven in equations (8),(9) and (10). Graphical representations in figure (2) and figure (3) are also valid for a viscid-compressible flow.

$$
\begin{gathered}
\Delta_{i}=\left(\frac{\nu}{y}\right)^{2}-2 \frac{q P_{A_{i}}}{\rho_{A_{i}}} \\
\Delta_{i-1}=\left(\frac{\nu}{y} \cos \left(\theta_{r}\right)\right)^{2}-2 \frac{r P_{A_{i-1}}}{\rho_{A_{i-1}}} \cos \left(\theta_{r}\right) \\
\overrightarrow{v_{i r}}=\frac{2 q P_{A_{i}} / \rho_{A_{i}}}{-\left(\frac{\nu}{y}\right) \pm \sqrt{\Delta_{i}}} ; \\
v_{i \overrightarrow{r-1}}=\frac{2 r P_{A_{i}} / \rho_{A_{i-1}}}{-\left(\frac{\nu}{y}\right) \pm \sqrt{\Delta_{i-1}}}
\end{gathered}
$$

The streamline shape theory satisfies corollary 4.1 for viscid-compressible flows. However, a significant difference exists in the resolved roots of equations (6) and (10). The roots of equation (10), $\overrightarrow{v_{i r}} \& \overrightarrow{v_{i r}-1}$ have pressure to density ratios in their numerators. Since the speed of sound of a fluid $c_{f l u}$ is the 
square root of the ratio of change in pressure and change in density as stated in equation (11).

$$
\begin{array}{r}
c_{f l u}=\sqrt{\frac{d P}{d \rho}} \\
\simeq \overrightarrow{v_{i r}}=\frac{2}{-\left(\frac{\nu}{y}\right) \pm \sqrt{\Delta_{i}}} \frac{q P_{A_{i}}}{\rho_{A_{i}}} \\
\simeq v_{i r-1}=\frac{2}{-\left(\frac{\nu}{y}\right) \pm \sqrt{\Delta_{i-1}}} \frac{r P_{A_{i}}}{\rho_{A_{i-1}}}
\end{array}
$$

As one of the roots obtain value closer to the speed of sound, the fluid flow doesn't form streamlines along the surface of the body. Equation (11) shows the limitation of streamline's shape theory. Speed of sound $c_{f l u}$ also equals to $\sqrt{\gamma r T}$. The effect of temperature on the viscosity and simultaneously on the formation of streamlines are in-evident in the streamline's shape theory. Therefore, this theory is only applicable to subsonic flows. Thus, streamline's shape theory satisfies the second corollary.

Corollary 4.2. At higher velocities, shock waves are formed rather than streamlines causing an abrupt change in the pressure as the body travels with velocity closer to the speed of sound.

\section{Results and Discussion}

The streamlines adopt the shape of the surface topology within the subsonic regime. These statements justly apply to only Newtonian fluids in a laminar flow. For fluid particles to alter their typical trajectory, the minimum velocity at a local point precisely is $|\nu / y|$. Here, the value of $\nu$ doesn't change with the change in the applied force at the local point, and $y$ represent the length of the boundary layer from the surface. As the velocity reaches closer to the speed of sound the fluid stream behaves like a wave. Thus, the hypothesis can't govern the phenomena of shock-wave formation.

The fluid stream interacts with a structured body at $A_{i-1}$, at $t_{0}$ when the impact of the surrounding area's interaction is non-existent. Equation (5) and equation (8) shows that one of the $\Delta_{i-1}$ is dependant on the value of $\theta_{r}$. This mathematically confirms that when a solid body interacts with a fluid stream. The leading edge where the fluid particles bombard on the surface $A_{i-1}$ at $t_{0}$, the fluid particles parallel in motion to the bombarded fluid particles haven't reached $A_{i}$, which contributes to non-existent of $\theta_{r}$. However, $A_{i-1}$ have already interacted with the surface of the body forming $\theta_{r}$ the resultant force vectors of $f\left(x_{1}\right)+g\left(x_{2}\right)$ will have the impact from $A_{i-1}$ at $t_{1}$. Thus fluid particles tend to travel along the surface of the body.Equations (8),(9) and (10) shows that viscid-compressible flow will have similar effects like inviscid-incompressible flows of a Newtonian fluid, regarding the impact of $\theta_{r}$ or the profile of the body. In other words, the shear stress influences 
cause the free stream to flow around the walls of the body by contributing to the streamline formation.

Here, $t_{0}$ is the time at which fluid particles reach the leading edge of the body at $A_{i-1} . t_{1}$ is the time at which fluid particles reach the subsequent point on the surface of the body $A_{i}$.

From figure 2, it is noticeable that the resultant function of the shear stress and dynamic pressure is a parabolic function with the change in the axis of symmetry. Here, the quadratic term's coefficient is $\frac{\rho}{2}$, and $\rho$ (density) is always positive for a fluid. As it is well-known if $\frac{\rho}{2}$ is greater than unity the parabola will be narrow, and the parabola shall open wider if the value is less than 1. As all viscous fluids will have positive viscosity the co-efficient of the linear term will also be positive. Thus, the axis of symmetry shifts to the left. Thus for non-Newtonian fluids or fluids with zero viscosity (super-fluids) can't be governed by the "Streamline's shape theory".

In figure 3 , which is the sum of two algebraic expressions $f\left(x_{1}\right)+g\left(x_{2}\right)$ the change in vertex. Figure 2 (a) is the graphical representation of the resultant function due to the flow-structure interaction at the stagnation point, where there is no effect on the fluid interactions of the surrounding area. Figure 2 (b) is the graphical representation of the resultant function due to the flow-structure interaction in due consideration of the effect of the fluid interactions of the surrounding area alone. Thus, it indicates how the shape of the body, the density, and viscosity of the fluid affects the flow-structure interactions by contributing to the decrement in the velocity of the flow at a particular point on the topology of the body. The change in vertex is due to a change in pressure, and these functions mentioned and illustrated in figure 2 causes a change in pressure. Thus, the vertex change is observable in figure 3. The narrowing of the parabola indicates a higher coefficient of the quadratic term, an indication of the compressibility effect. However, this model doesn't consider the effects of electromagnetic fields, thermal boundary layers, among many other aspects.

The Corollary 4.1 states that this model is mathematically appropriate for all inviscid-incompressible flows. In practice $\mathrm{M}<0.3$ (Mach number) is mostly considered as an inviscid-incompressible flow. But beyond $\mathrm{M}>0.3$ will be assumed as a viscous flow. The Corollary 4.2 indicates the mathematical limitation of this model which is observed in experimental results of existing literature. Tran-sonic flows defy the laws of low speed fluid dynamics. At higher velocities close to the speed of sound fluids exhibit wave characteristics unlike low speed flows.[13, 11]

From section 4 , it is evident that this theory distinguishes from other mathematical models in a few particular aspects.

1. Most of the earlier theories and their mathematical models based on experimental data emphasized on Buckingham- $\Pi$ theorem. This approach enabled the researchers by reducing the number of parameters to monitor during experimentation and made the process more convenient. However, this caused ignorance of a few parameters in the empirical equations 
when formulated. The shape of the body is one such parameter, which is often represented as correction in terms of coefficients.

2. The introduction of angle $\theta$, makes it possible to calculate the change in the flow along with the topology of the surface. If the direction of flow, shape of the body, and size of the body are known, then it will be possible to calculate the force at the stagnation point. Then from the stagnation point to the force vectors at succeeding locus of points are possible to be calculated at each corresponding time and space intervals.

3. In this formulation, the shape and size are inclusive in the fundamental equation. Trigonometric relations and finite element methods, coupled with this model, could make it a more reliable CFD solver.

4. Although this model doesn't consider Buckingham- $\Pi$ theorem, it is not resistant to $P i$ parameters. Once velocity is calculated at each point the surface of the body, through the historical data of $\mathrm{M}$ and Re (Reynolds number) various phenomena like flow transition, flow separation, the formation of shock-waves can be predictable on the topology of the body.

\section{Conclusion}

Hence, the stated hypothesis of the principal argument is satisfactorily proven, and the streamlines occur as a resultant of the shear stress and dynamic pressure distribution over the body.

The formation of streamlines and their mechanisms are the fundamental aspects that don't have a mathematical model in fluid dynamics. Although, many experts among various disciplines opine that this may not be of significant importance. But to develop more sophisticated CFD applications and methods of analysis, this is very critical.

The foremost advantage of Streamline's shape theory is that it is a lucid mathematical formulation and solutions always exist. Streamline's shape theory can evaluate various phenomena such as the Coand $\breve{a}$ effect, flow transition, flow separation, stalling, etc. Although other mathematical models can serve the same purpose, one needs different solvers and has to iterative simulations to compute various parameters.

Further course of possible research involves the evaluation of this theory utilizing experimentation methods and development of CFD solver based on the streamline's shape theory. A study is necessary to perceive the behavior of the non-Newtonian fluids concerning the topology of the body in flow-structure interactions and the formation of streamlines. 


\title{
REFERENCES
}

\section{Abbreviations}

\author{
CFD Computational Fluid Dynamics \\ M Mach number \\ Re Reynolds number \\ y length of boundary layer

\section{Nomenclature} \\ $\Delta \quad$ Discriminant of the quadratic equation \\ $\partial u / \partial y \quad$ Rate of shear deformation \\ $\mu \quad$ Dynamic viscosity of the fluid \\ $\nu \quad$ Kinematic viscosity of the fluid \\ $\rho \quad$ Density of the fluid \\ $\theta_{r} \quad$ Angle of resultant force on $A_{i}$ from preceding area \\ $A_{i-1}$ \\ $\vec{F} \quad$ Resultant force due to the fluid motion \\ $\vec{v} \quad$ Velocity of the fluid \\ $c_{f l u} \quad$ Speed of sound of the fluid \\ $d \rho \quad$ Change in the density \\ $d P \quad$ Change in the pressure \\ $P \quad$ Pressure at a local point \\ $\partial u / \partial y \quad$ rate of shear deformation

\section{Subscript} \\ $A_{i} \quad$ Local parameters such as $\rho, \mu, \vec{F}$ and $\vec{v}$ at the point \\ $\left(A_{i}\right)$ \\ $A_{i-1} \quad$ Local parameters such as $\rho, \mu, \vec{F}$ and $\vec{v}$ at the \\ preceding point $\left(A_{i}\right)$ \\ ir \& ir -1 Roots of the quadratic equations
}

\section{Acknowledgment}

I, Yuvaraj George, express my gratitude to all my colleagues, from Moscow Aviation Institute, who have extended their moral support and encouragement to carry out this work.

\section{Funding}

This research received no specific grant from any funding agency in the public, commercial, or not-for-profit sectors."

\section{References}

[1] WW Wood. Boundary layers whose streamlines are closed. Journal of Fluid Mechanics, 2(1):77-87, 1957.

[2] John Henry Michell. V. on the theory of free stream lines. Philosophical Transactions of the Royal Society of London.(A.), (181):389-431, 1890. 
[3] Akhil Datta-Gupta et al. Streamline simulation: A technology update (includes associated papers 71204 and 71764). Journal of petroleum technology, 52(12):68-84, 2000.

[4] $\mathrm{T} \mathrm{Wu}$. A free streamline theory for two-dimensional fully cavitated hydrofoils. 1955.

[5] Anatol Roshko. A new hodograph for free-streamline theory. 1954.

[6] GM Zaslavskii, RZ Sagdeev, and AA Chernikov. Stochastic nature of streamlines in steady-state flows. Zh. eksp. teor. Fiz, 94:102-15, 1988.

[7] CJ Farrugia, NV Erkaev, DF Vogl, HK Biernat, M Oieroset, RP Lin, and RP Lepping. Anisotropic magnetosheath: Comparison of theory with wind observations near the stagnation streamline. Journal of Geophysical Research: Space Physics, 106(A12):29373-29385, 2001.

[8] Tetsuo Suu. Dividing flow mechanisms in pipe junctions: Part 1-flow analysis based upon free-streamline theory and flow observation. Transactions of the Society of Heating, Air-conditioning and Sanitary Engineers of Japan, 3(7):51-62, 1978.

[9] Akhil Datta-Gupta and Michael Joseph King. Streamline simulation: theory and practice, volume 11. Society of Petroleum Engineers Richardson, 2007.

[10] Toshio Kobayashi and Kozo Kitoh. A review of cfd methods and their application to automobile aerodynamics. Technical report, SAE Technical Paper, 1992.

[11] LJ Clancy. Aerodynamics. new york: Jhon wiley and sons, 1975.

[12] Yuvaraj George. Mechanics of streamlines in an open circuit fluid flow and its impact on the flow. J. Adv. Res. Aero. Space Sci., 2019.

[13] John David Anderson. Modern compressible flow: with historical perspective, volume 12. McGraw-Hill New York, 1990.

[14] Yuvaraj George. Streamline's shape theory. Preprints, 2019.

[15] Daniel M Hanes and Douglas L Inman. Experimental evaluation of a dynamic yield criterion for granular fluid flows. Journal of Geophysical Research: Solid Earth, 90(B5):3670-3674, 1985.

[16] Herbert Amann. Linear and quasilinear parabolic problems, volume 1. Springer, 1995.

[17] Nitsa Movshovitz-Hadar. A constructive transition from linear to quadratic functions. School Science and Mathematics, 93:288-288, 1993.

[18] David E Muller. A method for solving algebraic equations using an automatic computer. Mathematical tables and other aids to computation, 10(56):208-215, 1956.

Yuvaraj George

Kafedra 101, Faculty No.1-School of Aeronautics

Moscow Aviation Institute

4, Volokolamskoe Shosse

Moscow, 125993 Russian Federation

e-mail: georgeyuvaraj2@gmail.com 